\title{
The Clinical Benefits, Limitations, and Perspectives of the ABC Method
}

\author{
Hiroshi Kishikawa \\ Key words: gastric cancer, Helicobacter pylori, pepsinogens, cancer screening, ABC method, atrophic \\ gastritis
}

(Intern Med 59: 1471-1472, 2020)

(DOI: 10.2169/internalmedicine.4450-20)

In Japan, over $99 \%$ of gastric cancer cases are associated with Helicobacter pylori infection (1). Because the grade of $H$. pylori-induced gastric atrophy correlates with gastric cancer development (2) and the serum pepsinogen (PG) levels reflect gastric mucosal atrophy, the combination of the PG test and the evaluation of the H. pylori antibody titer, a serological screening approach called the "ABC method" or "ABC classification," efficiently and inexpensively stratifies the risk of gastric cancer in patients without a history of eradication (3).

The ABC method classifies patients into group A $[H . p y-$ lori(-), PG(-)], H. pylori-negative; group B [H. pylori(+), PG $(-)]$, H. pylori infection without extensive chronic atrophic gastritis (CAG); group C [H. pylori $(+), \mathrm{PG}(+)]$, H. pyloriinduced extensive CAG; and group D [H. pylori(-), $\mathrm{PG}(+)]$, spontaneous disappearance of $H$. pylori due to severe CAG. This method can detect serologically negative but H. pyloriinfected patients (group D) and thus is more accurate than H. pylori serology alone. Group B, C and D include candidates for endoscopy (3), and the cancer detection rate of the $\mathrm{ABC}$ method is $0.22 \%-0.42 \%$, which is higher than historical data obtained by conventional X-ray mass screening $(0.15 \%)$ (4). In the present report, the clinical benefits, limitations, and perspectives of the ABC method are discussed.

Few high-risk cases with gastric atrophy and gastric cancer are misclassified into group A, which is the "false group A problem" and regarded as the greatest weakness of the ABC method (5). Most of these patients have "past infection-induced atrophic gastritis" after normalization of PG and $H$. pylori serology caused by unintentional eradication through the incidental use of antibiotics (6). To avoid this misclassification, the cut-off value of the H. pylori antibody titer is set at a low value. In 2015, the Ministry of Health, Labor, and Welfare recommended population-based radiographic and endoscopic screenings for gastric cancer; however, the $\mathrm{ABC}$ method was not recommended because of the lack of direct evidence of mortality reduction. The high rate of patients for whom endoscopy is indicated (more than that in group B) is the other weakness of the ABC method, which requires improvement in the future.

In this issue of Internal Medicine, Kishino et al. reported that about $6.0 \%(364 / 6,105)$ of group A patients showed open-type gastric atrophy. The detection rates of gastric cancer in groups A, B, C, and D were $0.07 \%(4 / 6,105), 0.5 \%$ $(8 / 1,739), 0.8 \%(16 / 2,010)$, and $1.1 \%(3 / 281)$, respectively. No gastric cancer was recognized among endoscopically normal cases $(0 / 4,567)$; they therefore suggested that an evaluation by endoscopy is superior to the serological screening method (7).

Is the $\mathrm{ABC}$ method a relic of gastric cancer screening? No, it has clinical significance, and whether or not the mortality rate decreases when using this screening method should be determined. The benefits of the ABC method are its cost effectiveness and high sensitivity rate. As Kishino et al. reported, its sensitivity for detecting gastric cancer was $87 \%$ (27/31) (7), which is better than the rate of the immunological fecal occult blood test (65.8\%) (8). Henceforth, the rate of gastric cancer is expected to significantly decrease because the infection rate of $H$. pylori has decreased (9). Intensive detection and monitoring of $H$. pyloripositive cases is effective, and the $\mathrm{ABC}$ method will become more useful, especially in populations with low $H$. pylori infection rates.

In addition, the $\mathrm{ABC}$ method can be used in various clinical settings, especially in east Asian countries in the following instances: 1) to detect high-risk cases if its limitations are recognized, and 2) to determine the $H$. pylori infection status and risk of future gastric cancer development for patients who refuse endoscopy or have undergone endoscopy. Furthermore, it motivates patients to undergo endoscopy, en- 
ables the potential eradication of $H$. pylori in positive cases, and provides information on the appropriate frequency of endoscopy in each case. Considering the decreasing infection rate of $H$. pylori and consequent decreasing incidence of gastric cancer in Japan, the clinical significance of the $\mathrm{ABC}$ method may become more important in the future.

The author states that he has no Conflict of Interest (COI).

\section{References}

1. Matsuo T, Ito M, Takata S, Tanaka S, Yoshihara M, Chayama K. Low prevalence of Helicobacter pylori-negative gastric cancer among Japanese. Helicobacter 16: 415-419, 2011.

2. Correa P. Human gastric carcinogenesis: a multistep and multifactorial process-First American Cancer Society Award Lecture on Cancer Epidemiology and Prevention. Cancer Res 52: 6735-6740, 1992.

3. Miki K. Gastric cancer screening by combined assay for serum anti-Helicobacter pylori $\mathrm{IgG}$ antibody and serum pepsinogen levels - "ABC method". Proc Jpn Acad Ser B Phys Biol Sci 87: 405414, 2011.

4. Yamaguchi Y, Nagata Y, Hiratsuka R, et al. Gastric cancer screen- ing by combined assay for serum anti-Helicobacter pylori $\operatorname{IgG}$ antibody and serum pepsinogen levels-the ABC method. Digestion 93: 13-18, 2016.

5. Kishikawa H, Kimura K, Ito A, et al. Cutoff pepsinogen level for predicting unintendedly eradicated cases of Helicobacter pylori infection in subjects with seemingly normal pepsinogen levels. Digestion 95: 229-236, 2017.

6. Kishikawa H, Ojiro K, Nakamura K, et al. Previous Helicobacter pylori infection-induced atrophic gastritis: a distinct disease entity in an understudied population without a history of eradication. Helicobacter 25: e12669, 2020.

7. Kishino T, Oyama T, Tomori A, et al. Usefulness and limitations of a serum screening system to predict the risk of gastric cancer. Intern Med 59: 1473-1480, 2020.

8. Morikawa T, Kato J, Yamaji Y, et al. A comparison of the immunochemical fecal occult blood test and total colonoscopy in the asymptomatic population. Gastroenterology 129: 422-428, 2005.

9. Mizota Y, Yamamoto S. How long should we continue gastric cancer screening? From an epidemiological point of view. Gastric Cancer 22: 456-462, 2019.

The Internal Medicine is an Open Access journal distributed under the Creative Commons Attribution-NonCommercial-NoDerivatives 4.0 International License. To view the details of this license, please visit (https://creativecommons.org/licenses/ by-nc-nd/4.0/).

(C) 2020 The Japanese Society of Internal Medicine Intern Med 59: 1471-1472, 2020 